\title{
Molecular characterization of a glycerol-3-phosphate acyltransferase reveals key features essential for triacylglycerol production in Phaeodactylum tricornutum
}

\author{
Ying-Fang $\mathrm{Niu}^{1,2+}$, Xiang Wang ${ }^{1 \dagger}$, Dong-Xiong Hu${ }^{1}$, Srinivasan Balamurugan ${ }^{1}$, Da-Wei Li ${ }^{1}$,
} Wei-Dong Yang ${ }^{1}$, Jie-Sheng Liu' ${ }^{1}$ and Hong-Ye Li ${ }^{*}$

\begin{abstract}
Background: The marine diatom, Phaeodactylum tricornutum, has become a model for studying lipid metabolism and its triacylglycerol (TAG) synthesis pathway makes it an ideal target for metabolic engineering to improve lipid productivity. However, the genetic background and metabolic networks of fatty acid biosynthesis in diatoms are not well understood. Glycerol-3-phosphate acyltransferase (GPAT) is the critical enzyme that catalyzes the first step of TAG formation. So far, characterization of GPAT in marine microalgae has not been reported, especially at the level of comprehensive sequence-structure and functional analysis.

Results: A GPAT was cloned from P. tricornutum and overexpressed in P. tricornutum. Volumes of oil bodies were produced and the neutral lipid content was increased by twofold determined by Nile red fluorescence staining. Fatty acid composition was analyzed by GC-MS, which showed significantly higher proportion of unsaturated fatty acids compared to wild type.

Conclusion: These results suggested that the identified GPAT could upregulate TAG biosynthesis in P. tricornutum. Moreover, this study offers insight into the lipid metabolism of diatoms and supports the role of microalgal strains for biofuels production.
\end{abstract}

Keywords: GPAT, Lipid, Diatoms, Biofuels

\section{Background}

Renewable energy is one of the most effective solutions to address the carbon emission, energy security, and increased fuel consumption challenges that result in global warming and fossil fuel price concerns. These issues have prompted intensive interest in the capability of oleaginous microalgae to generate renewable oil sources which can be readily converted into biodiesel [1]. Microalgae can accumulate oil and are considered

\footnotetext{
${ }^{*}$ Correspondence: thyli@jnu.edu.cn

${ }^{\top}$ Ying-Fang Niu and Xiang Wang contributed equally to this work

${ }^{1}$ Key Laboratory of Eutrophication and Red Tide Prevention

of Guangdong Higher Education Institutes, College of Life Science,

Jinan University, Guangzhou 510632, China

Full list of author information is available at the end of the article
}

to be a promising feedstock for renewable biofuel production. For example, microalgae demonstrate much higher biomass productivity compared to higher plants and algal growth facilities could potentially be located in aquatic environments, which will not increase arable land concerns.

Although microalgae have reemerged as a potential 3rd generation feedstock for biofuel production, large-scale harvesting of microalgae is hampered by the lack of algal strains that can be selectively optimized for both high biomass generation capability and high TAG content [2]. One potential solution is to engineer robust oil-yielding microalgae by expressing the critical enzymes for TAG accumulation and to acquire a better overall understanding of lipid metabolic pathways in microalgae $[3,4]$. 
In eukaryotes, TAGs are identified as neutral lipids that serve as the crucial storage form of energy. TAGs are the major feedstock for biodiesel production. TAG accumulation in microalgae is usually correlated to environmental stresses, such as high light intensity, high temperature, nitrogen limitation, and salinity $[5,6]$. There are three major steps involved in TAG synthesis. Firstly, carboxylation of acetyl-CoA to form malonyl-CoA, which is the committing step of fatty acid biosynthesis in the plastid, secondly, acyl chain elongation in the plastid and cytosol, and finally, TAG formation in the endoplasmic reticulum (ER) [4]. The biosynthesis of fatty acids in chloroplast is catalyzed by two major, evolutionarily conserved enzymes, acetyl-CoA carboxylase (ACCase) and fatty acid synthase (FAS). The synthesized fatty acids are then esterified by glycerol-3-phosphate acyltransferase (GPAT) to glycerol 3-phosphate at the sn-1 position to form lysophosphatidic acid (LysoPA) [5, 7]. LysoPA is further catalyzed into phosphatidic acid (PA) by lysophosphatidic acid acyltransferase (LPAAT). The PA is then dephosphorylated by phosphatidic acid phosphatase (PAP) to form diacylglycerol (DAG). It has been reported that PA and DAG can also be formed in the chloroplast, where they serve as precursors for the synthesis of structural membrane lipids and neutral lipids $[8,9]$.

GPAT (EC 2.3.1.15) is considered as the initial enzyme for glycerolipid synthesis. In mammals, four GPAT isoforms have been identified [10-12]. In rats, hepatic glycerol-sn-3-phosphate acyltransferase 1 was overexpressed and caused hepatic insulin resistance, suggesting a role for lipid metabolites in the development of insulin resistance [7]. In humans, multiple isoforms of GPAT were expressed and differentially regulated in epidermis/ keratinocytes [13]. In the model dicot plant, Arabidopsis thaliana, ten GPAT isoforms have been reported recently $[14,15]$. These ten genes can be divided into three clusters. The first cluster is plastid-localized GPAT, which uses acyl-ACP substrates, and exhibits sn-1 acyl transfer regiospecificity [16]. The second cluster is GPAT9, which is located in the endoplasmic reticulum, and is enable to synthesize non-plastid glycerolipid [17]. The remaining eight GPATs do not play a role in the Kennedy pathway $[14,17]$. Daubossy et al. reported that disruption of the UDP-glucose pyrophosphorylase gene in Phaeodactylum tricornutum resulted in increased TAG accumulation [18]. In our previous report, we successfully developed transgenic $P$. tricornutum with increased lipid accumulation by overexpressing type 2 diacylglycerol acyl transferase (DGAT) [19]. Similarly, overexpression of DGAT in $P$. tricornutum resulted in increased proportion of polyunsaturated fatty acids [20, 21]. However, no GPAT has been characterized in microalgae. Report of research employing metabolic engineering to increase lipid productivity in microalgae has been limited to date. In the present study, we first cloned a putative GPAT from the oleaginous marine diatom, P. tricornutum, and characterized its biological functions. This study will enable the use of genetically improved microalgal strains for industrial biofuel production.

\section{Results and discussion \\ Analysis of GPAT sequences}

Conserved amino acid sequences for GPAT orthologs from various species were cataloged and used for BLAST interrogation of the P. tricornutum genome (National Center for Biotechnology Information-NCBI, http:// www.ncbi.nlm.nih.gov/). Only one putative GPAT (PHATRDRAFT_42446, annotated as hypothetical protein) with high similarity to the consensus of GPAT was retrieved. Interestingly, PHATRDRAFT_42446 was annotated as a hypothetical protein and 1-acylglycerol-3-phosphate $O$-acyltransferase [EC:2.3.1.51] in KEGG (http://www.genome.jp/kegg/). The 315-amino acid sequence of this PtGPAT was predicted to contain a LPLAT_AGPAT-like domain spanning amino acid 100-292 (Fig. 1a), with a putative acyl-acceptor binding pocket predicted by a BLAST conserved domains search (http://www.ncbi.nlm.nih.gov/Structure/cdd/wrpsb.cgi). The phylogenetic relationships of GPAT with orthologs from various species were analyzed with the MEGA5 software using the neighbor-joining method. As shown in Fig. 1b, GPAT orthologs from 24 species were clustered into three groups. GPAT from P. tricornutum was closely aligned with orthologs from the marine diatoms, Thalassiosira pseudonana and Thalassiosira oceanica. Moreover, they were clustered into the same group with the marine brown alga, Ectocarpus siliculosus. This group was separated from the other two groups consisting of micro- and macroalgal origins. These results suggest that PtGPAT was well conserved during evolutionary development, having close relationships with other diatoms and brown algae, while remaining far from other species.

\section{Construction of transformation vector and generation of transgenic $P$. tricornutum}

PtGPAT cDNA was fused between the fucoxanthin chlorophyll a/c binding protein (fcp) fcpC promoter and fcpA terminator of $P$. tricornutum. Furthermore, an Omega leader sequence was added before the coding region of PtGPAT for enhancing its translation (Fig. 2a). A c-Myc tag was also fused to the $C$-terminal of PtGPAT. Hence, the expression of heterologous PtGPAT could be followed by Western blot analysis using anti-Myc antibody. CAT (chloramphenicol acetyltransferase) was used as selection marker gene. After electroporation, transformed algal cells were selected and grown for at least four successive 


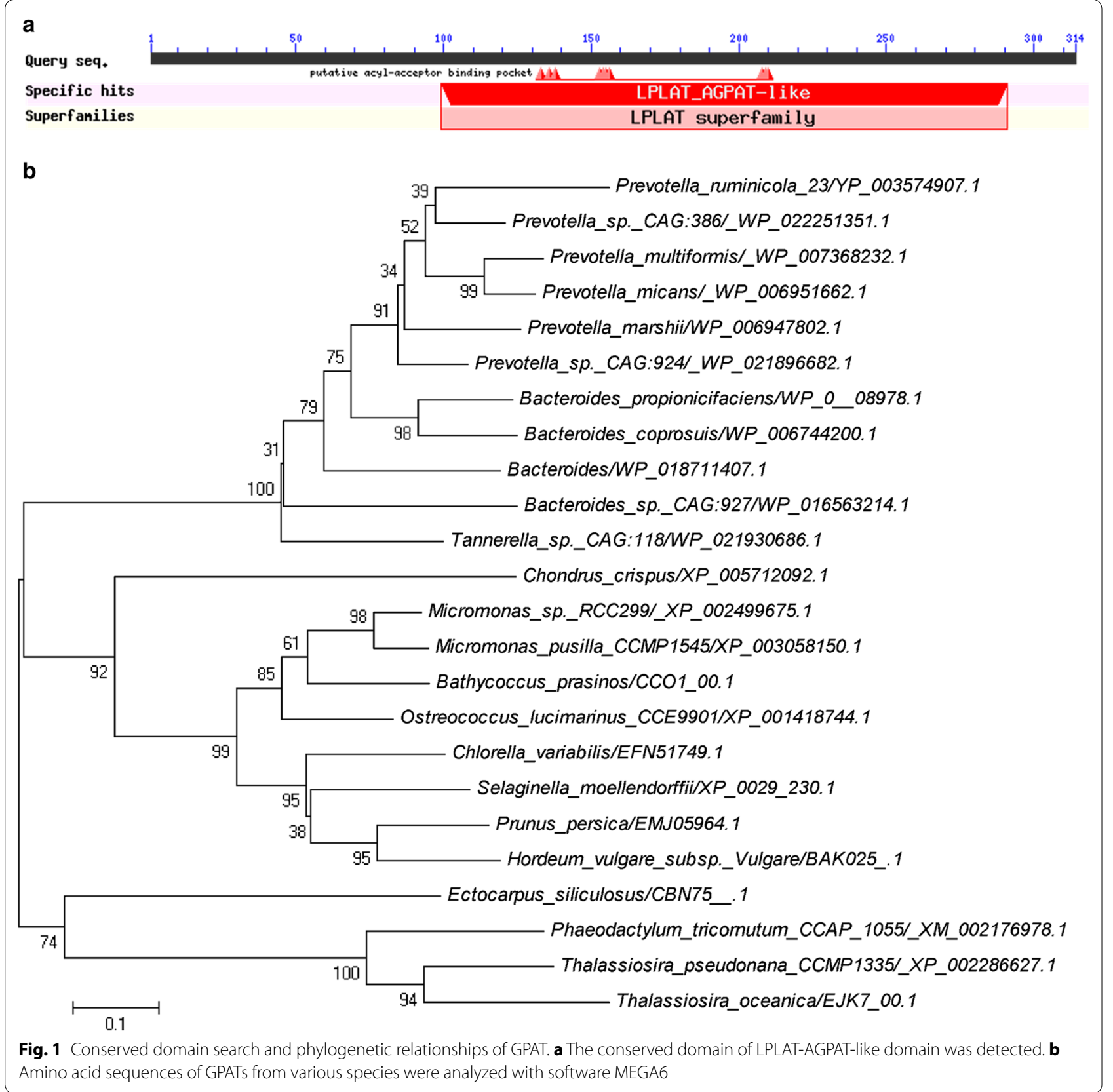

subculture cycles under chloramphenicol $\left(250 \mu \mathrm{g} \mathrm{mL} \mathrm{m}^{-1}\right)$ selection followed by molecular characterization. The integration of the PtGPAT expression cassettes in the transformed $P$. tricornutum cells was confirmed by single-cell PCR using CAT primers. An expected PCR product of $0.7-\mathrm{kb}$ PCR was detected in the transgenic microalgae, whereas no band was present in the wildtype strain (data not shown). These results suggested that the expression vector was successfully introduced into P. tricornutum. Several independent transgenic cell lines have been initially screened in terms of growth and neutral lipid content. To consider the performance of integrated characteristics, only the most remarkable transgenic line was discussed in the following sessions.

The relative transcript level of GPAT in transgenic lines was determined by $\mathrm{qPCR}$ using $\beta$-actin as a reference gene. Transcript abundance of GPAT was significantly increased by 3.7-fold in the selected transgenic line compared to wild type (Fig. 2b). To confirm the expression of PtGPAT protein, Western blot analysis was performed 


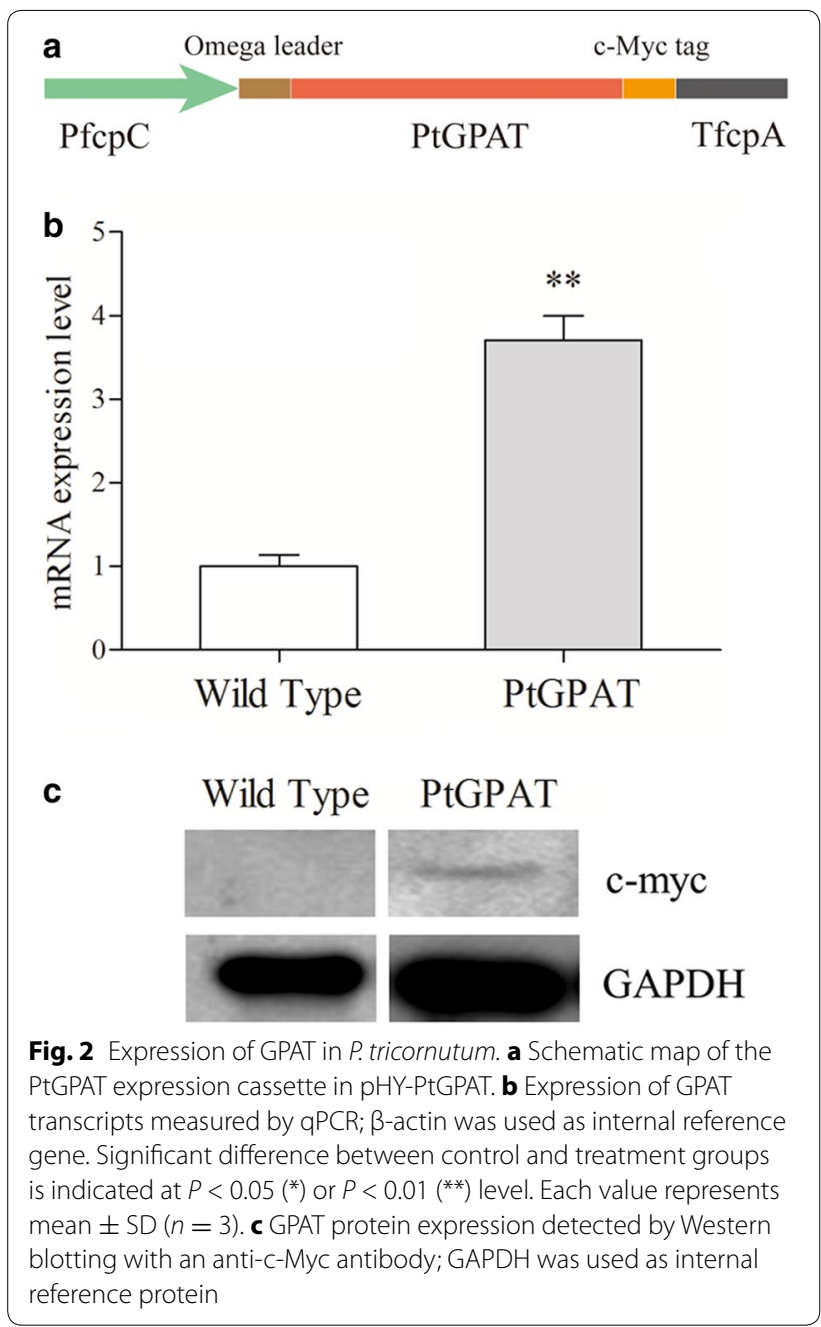

with an anti-c-Myc antibody. A specific cross-reacting band in accordance with the expected molecular weight was detected in the transgenic cells, whereas no analogous band was observed in the wild-type strains (Fig. 2c), indicating that the introduced PtGPAT was transcribed and also translated in the transgenic cells.

\section{Growth characteristics and photosynthetic performance of transgenic microalgae}

Photosynthesis activity was measured to indicate the photosynthetic performance and acclimation status of transgenic $P$. tricornutum. The chlorophyll fluorescence parameter, $\mathrm{Fv} / \mathrm{Fm}$, was monitored for algal cultures in the stationary phase (Fig. 3a). Fv/Fm is the maximum photochemical quantum yield of PSII reaction center to reflect the light energy conversion efficiency of photosynthesis $[22,23]$. A significant increase in $\mathrm{Fv} / \mathrm{Fm}$ was detected in transgenic microalgae compared to wild type. It has been reported that the unsaturation of membrane fatty acids by the action of GPAT could result in the protection of

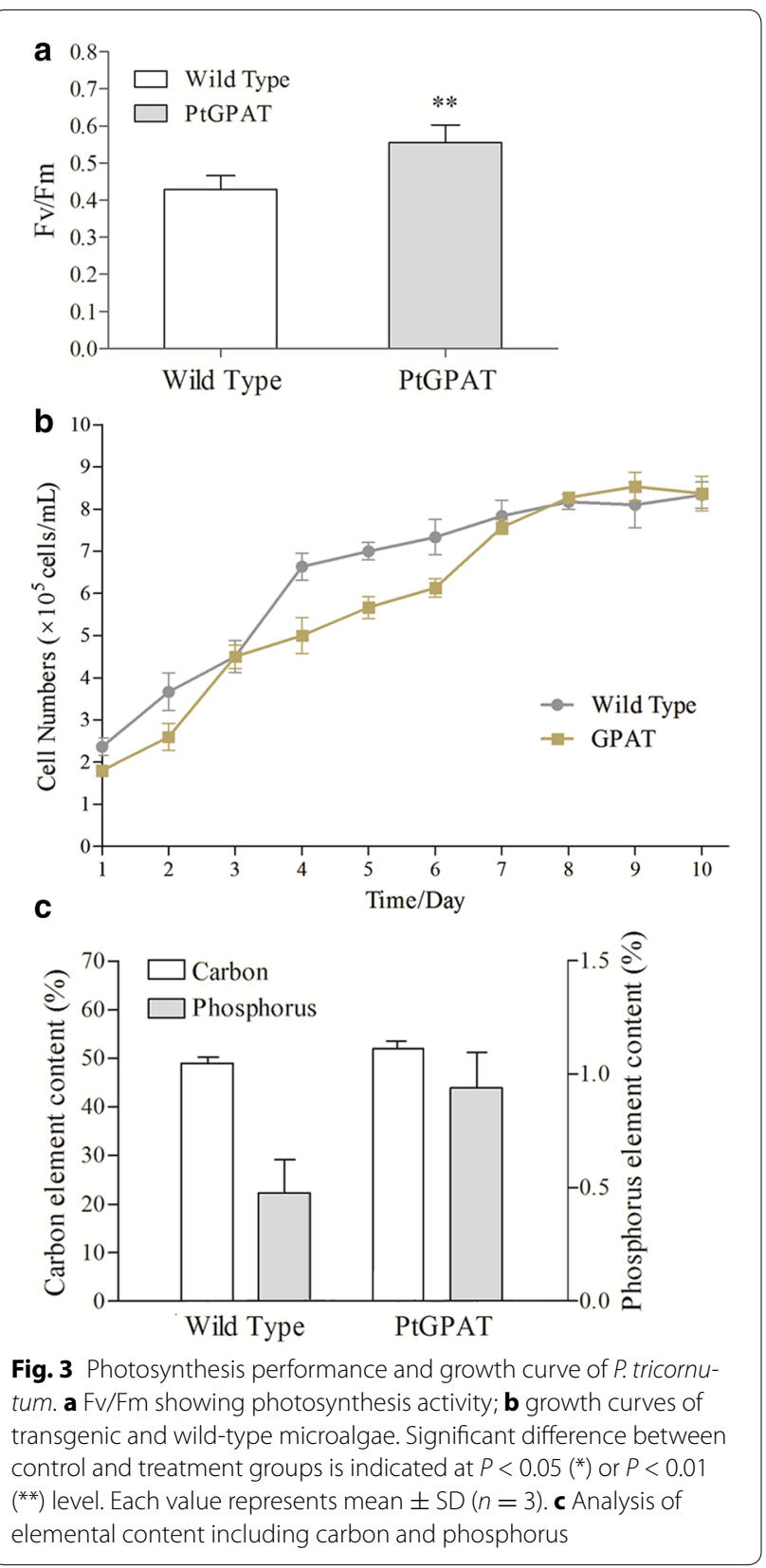

the photosynthetic mechanism from photo-inhibition in tobacco and cyanobacteria [24].

In order to correlate the growth characteristics with the GPAT overexpression, we analyzed the growth curve for both transgenic and wild-type cells. As shown in Fig. 3b, transgenic and wild-type cells showed only a slight variation in growth curve and essentially reached stationary phase in a similar way. The results suggested that the GPAT overexpression did not have an apparent effect on the growth and biomass accumulation of transgenic microalgae. 
Elemental analysis of carbon and phosphorus in P. tricornutum were further performed. As shown in Fig. 3c, transgenic cells showed a slightly higher carbon content than wild type. This increase of carbon content correlated well with the elevated carbon assimilation reflected by the higher photosynthetic performance. Phosphorus is the major constituent of the phospholipids which comprise cellular membranes. Up to a twofold increase in phosphorus was detected in transgenic microalgae. This increase in phosphorus content could reside in the phospholipids which form the membranes of oil bodies increased in transgenic cells. In some microalgal species, phospholipids in plastidial membranes could be recycled through membrane remodeling and used as source material for storage neutral lipid synthesis during certain stress conditions, such as nitrogen limitation $[25,26]$.

\section{Lipid productivity analysis}

Neutral lipid content was first determined by Nile red staining in the stationary phase. Transgenic microalgae increased twofold compared to wild type (per $10^{6}$ cells) in the stationary phase (Fig. 4a). The neutral lipid content in dry cell weight (DCW) was further determined by conventional gravimetric measurements. The DCW per $10^{9}$ transgenic cells was measured to be $30.53 \mathrm{mg}$ and the lipid yield was $12.21 \mathrm{mg}$. Correspondingly, the neutral lipid content was $42.6 \%$ (DCW) in the transgenic microalgae, whereas it was $25.5 \%$ in wild type (Fig. $4 \mathrm{~b}$ ). In terms of industrial production, the lipid productivity per culture volume is an important factor. As shown in Fig. 4c, wild-type cells were determined to contain a total lipid content of $480 \mathrm{mg} \mathrm{L}^{-1}$ culture, whereas transgenic cells reached $810 \mathrm{mg} \mathrm{L}^{-1}$ culture in the stationary phase. These results indicated that GPAT overexpression markedly improved the lipid productivity in diatom cells.

Since nitrogen deprivation can stimulate lipid production in some microalgae species, diatom cells in the stationary phase (7 days after subculture) were also subjected to nitrogen-deprivation treatments for $48 \mathrm{~h}$. As shown in Fig. 4d, upon nitrogen-deprivation treatment, the neutral lipid content increased by 2.45 -fold in the wild type and by 1.59 -fold in the transgenic cells. Thus, a

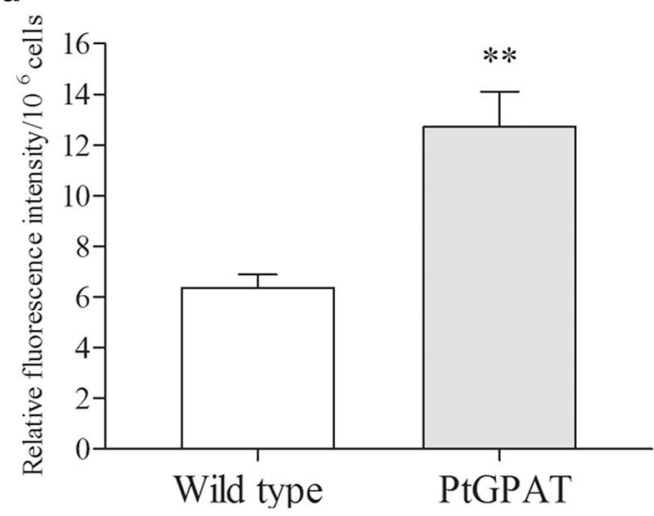

c

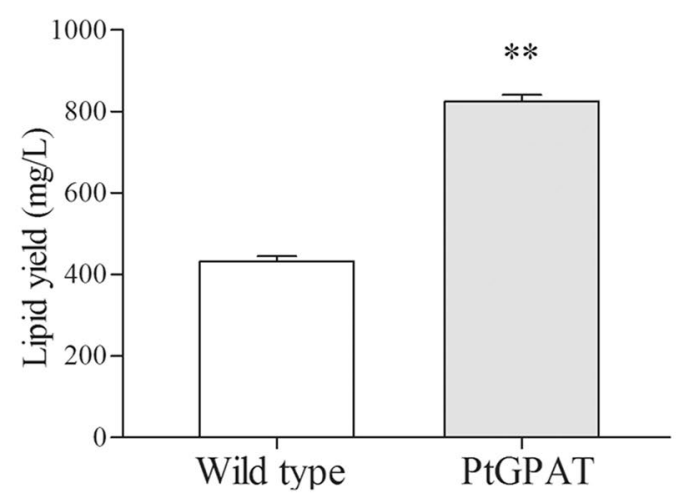

b

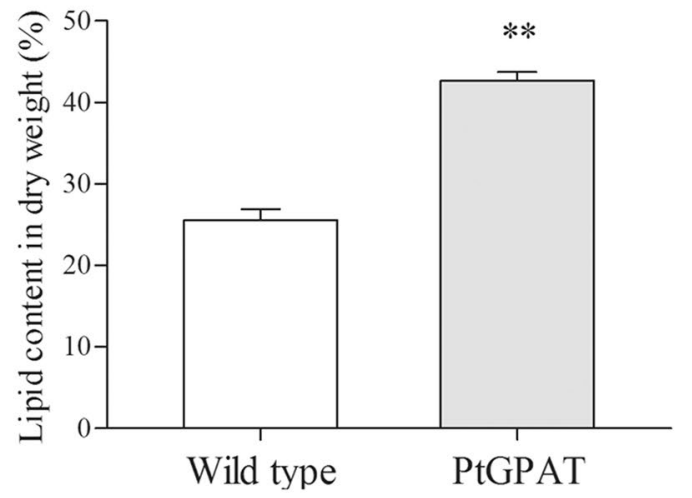

d

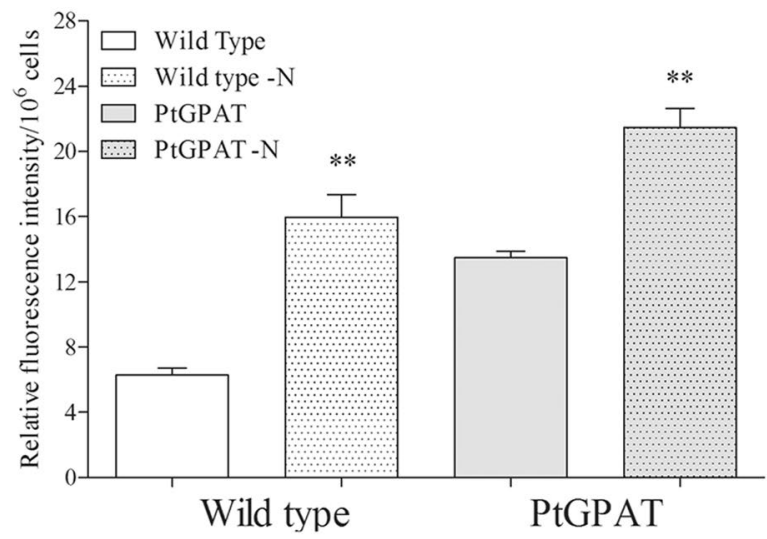

Fig. 4 Lipid productivity in P. tricornutum. a Neutral lipid content as per $10^{6}$ cells determined by Nile red staining. b Total lipid content in dry cell weight determined by gravimetry. c Total lipid content as per culture volume. $\mathbf{d}$ Neutral lipid content under N-deprivation. Significant difference between control and treatment groups is indicated at $P<0.05\left(^{*}\right)$ or $P<0.01\left(^{* *}\right)$ level. Each value represents mean $\pm \operatorname{SD}(n=3)$ 
the final neutral lipid content in transgenic cells was 1.34fold that of the wild type.

To further examine the lipid accumulation in diatom cells, oil bodies were observed by Nile red staining under a confocal microscope. As shown in Fig. 5, the numbers of oil bodies were similar in both transgenic and wildtype cells, whereas the volume of oil bodies in transgenic cells was considerably increased and larger than in wild type. The larger volume of oil bodies that appeared in $P$. tricornutum overexpressing GPAT was consistent with the increase in neutral lipid content observed.

\section{Changes in fatty acid composition regulated by overexpression of GPAT}

In order to detect the effect of GPAT overexpression on fatty acid composition in transgenic and wild-type microalgae, cells in the stationary phase were analyzed by GC-MS analysis. Interestingly, significant differences in fatty acid composition were detected between transgenic and wild-type cells (Table 1). Proportions of saturated fatty acids (SFA) and monounsaturated fatty acids (MUFA) decreased by 35 and $12 \%$, respectively; but polyunsaturated fatty acids (PUFA) increased by $41 \%$. Notably, C16:3 fatty acid was $52 \%$ higher than that of wild-type cells, and C20:5 was almost $40 \%$ higher; however, C16:0 was about $45 \%$ lower. The changes in fatty acid composition were largely due to dramatic increases in PUFAs, which are generally the abundant fatty acids in P. tricornutum [27]. These increases correlate with losses of SFAs and MUFAs. Thus, the altered content of unsaturated fatty acids in transgenic diatoms was due to the activity of overexpressed GPAT. Yokoi et al. reported that expression of Arabidopsis GPAT resulted in increased content of unsaturated fatty acids in transgenic rice compared to wild type [28]. Expression of Brassica napus GPAT resulted in increased oil content in transgenic tobacco [29]. Iskandarov et al. reported that fatty acid content was increased in the transgenic green microalga, Chlamydomonas reinhardtii, expressing Lobosphaera incisa GPAT and resulted in increased TAG accumulation [30]. Similarly, induced expression of Helianthus annuus GPAT in transgenic Escherichia coli resulted in increased unsaturated fatty acid content [31]. Accordingly, the proportion of unsaturated fatty acids showed
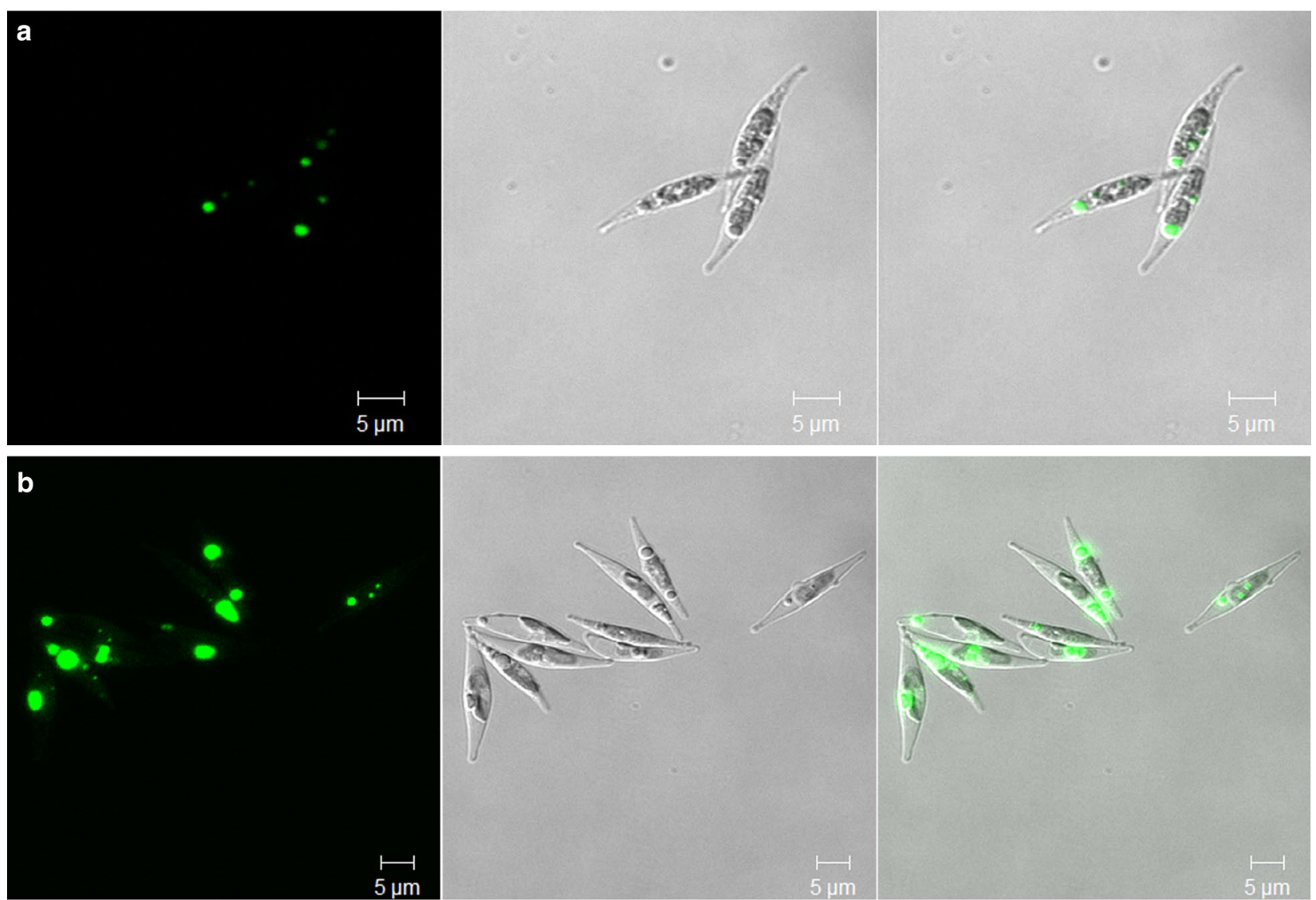

Fig. 5 Morphological observations of the cell and oil bodies. Microalgal cells were stained with Nile red and observed under a laser-scanning confocal microscope. a wild type; b transgenic cells. Left panel fluorescence of oil bodies; middle panel differential interference contrast (DIC); right panel overlay image. Bar $=5 \mu \mathrm{m}$ 
Table 1 Changes in fatty acid composition in transgenic and wild-type microalgae

\begin{tabular}{lcc}
\hline Fatty acid & $\begin{array}{l}\text { Wild type } \\
\text { (DW \%) }\end{array}$ & $\begin{array}{c}\text { GPAT } \\
\text { (DW \%) }\end{array}$ \\
\hline C14:0 & 3.54 & 2.48 \\
C15:0 & 0.20 & 0.11 \\
C16:0 & 23.60 & 16.17 \\
C18:0 & 7.35 & 5.93 \\
C22:0 & 0.17 & 0.36 \\
C24:0 & 0.29 & 0.85 \\
SUM SFA & 35.15 & 25.9 \\
C16:1 & 25.08 & 26.85 \\
C18:1 & 5.95 & 0.84 \\
SUM MUFA & 31.03 & 27.69 \\
C16:3 & 4.19 & 6.41 \\
C18:2 & 1.09 & 1.66 \\
C18:3 & 0.08 & 0.04 \\
C20:5 & 18.23 & 25.53 \\
C22:6 & 0.81 & 0.87 \\
SUM PUFA & 24.4 & 34.51 \\
\hline
\end{tabular}

an increase in the present study. For the industrial scale biofuel production process, an optimized biological source is a prerequisite. The transgenic marine diatom overexpressing GPAT reported here could be a promising biofuel source with high lipid content and unsaturated fatty acids.

\section{Subcellular localization of GPAT in P. tricornutum}

PtGPAT was predicted to consist of two transmembrane helices by membrane topology prediction server HMMTOP (http://www.enzim.hu/hmmtop/server/ hmmtop.cgi), spanning amino acid 9-23 and 56-80. Subcellular localization analysis was performed using prediction programs. A signal peptide was predicted in PtGPAT using TargetP 1.1 Server (http://www.cbs.dtu. $\mathrm{dk} /$ services/TargetP-1.1/) with a 0.754 possibility. WoLF PSORT Prediction (http://www.genscript.com/psort/ wolf_psort.html) was used to further predict the subcellular localization, which suggested that the most likely location was the chloroplast. LocTree3 (https://rostlab. org/services/loctree3) also predicted the chloroplast location.

The subcellular localization of PtGPAT in diatom cells was further determined by immuno-electron microscopy. Gold particles were found predominantly in chloroplasts in PtGPAT-overexpressing transgenic cells (Fig. 6a, b), whereas no gold particles could be found in the wild type (Fig. 6c). These results indicated that PtGPAT was predominantly localized in the chloroplast, which is overall consistent to the software prediction.

Subcellular localization is a crucial attribute of a protein and indispensable to understanding the biological processes at the cellular level. Hence, predicted subcellular localization of proteins is fundamental to Systems Biology [32]. So far, the subcellular localizations of PtGPAT were determined to be varied in different organisms, including the cytosol, chloroplasts, or mitochondria. In 1988, cDNA of GPAT was first cloned in chloroplasts of squash cotyledon [33]. Some algal and higher plant GPAT proteins were also predicted in chloroplast using subcellular targeting localization prediction [5]. In sunflowers, abundant sn-glycerol-3-phosphate acyltransferase was identified in the chloroplast [31]. Subcellular localization prediction analysis in the arachidonic acid-rich microalga, Myrmecia incisa Reisigl H4301, demonstrated that
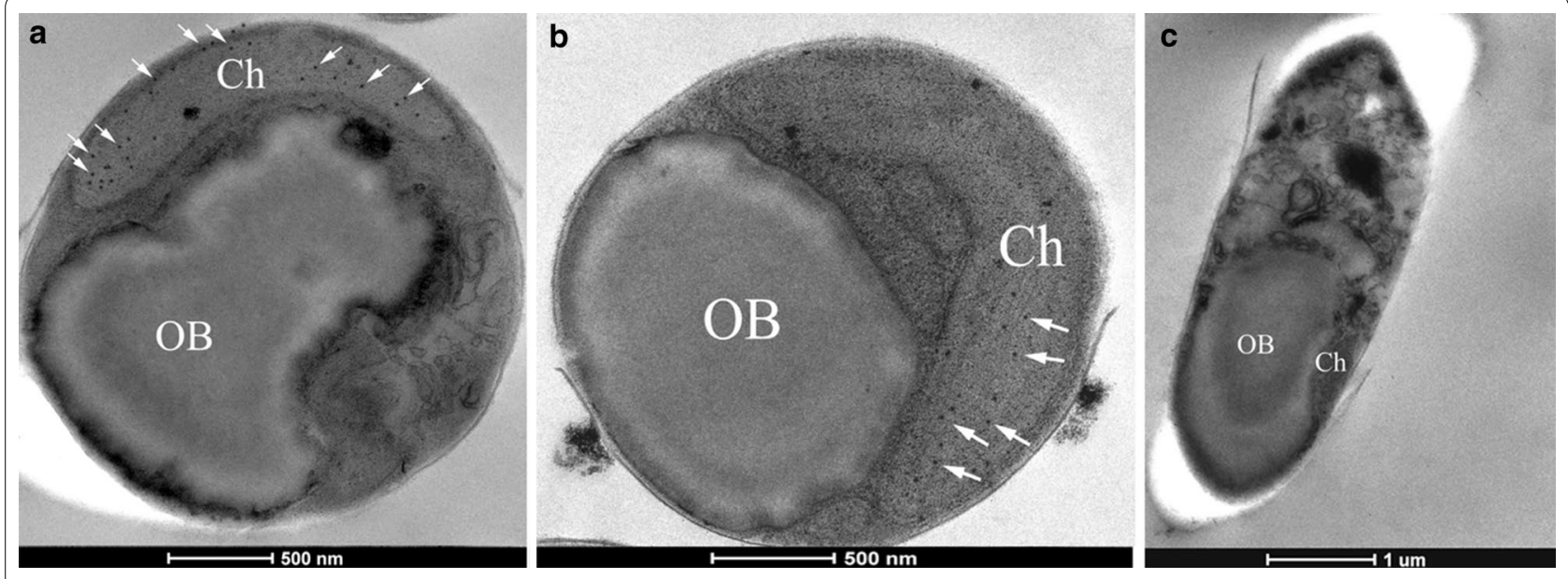

Fig. 6 Subcellular localization of GPAT in P. tricornutum. The Myc-tagged PtGPAT was detected by immuno-gold labeling with Myc-specific antibody in the microalgal cell. Transverse sections of transgenic cell (a and $\mathbf{b})$ and wild type (c) were photographed under EM. The arrows indicate the gold particles; $O B$ oil body; Ch chloroplast; Bars $=500 \mathrm{~nm}$ in (a and b) and $1 \mu \mathrm{m}$ in (c) 
GPAT might target the chloroplast [34]. Two glycerol3-phosphate acyltransferase-like (GPAT-like) proteins from the tung tree, GPAT8 and GPAT9, were found to co-localize in the same ER subdomains [35]. Functional differences between ER-GPAT and mitochondrial (Mt)GPAT in Caenorhabditis elegans demonstrated that MtGPAT was required for mitochondrial fusion [36].

In this study, GPAT was predicted to localize in the chloroplast and this notion was evidenced by immunoEM. In photosynthetic organisms, fatty acid biosynthesis can take place in the chloroplast. GPAT, along with the acyl-(acyl carrier protein), catalyzes the first reaction of glycerolipid assembly in the chloroplast; the substrates for these enzymes are synthesized within the chloroplast [31]. By a series of sequential enzymatic reactions, once the fatty-acyl chain attains its chain length (C 16:0 or C 18:0), it can be esterified by GPAT to form LysoPA. LysoPA is added to subsequent acyl chains from LPAAT to give PA, which is dephosphorylated to yield DAG, the precursor for algal glycerolipids [5, 37, 38]. The free fatty acids from the plastid are transported to the chloroplast and ER, where they are attached to -CoA by long-chain acyl-CoA synthase. The same sequential reactions can occur in the microalgal chloroplast [37, 38].

\section{Conclusions}

Considering that functional biochemical studies of key enzymes in microalgae have been lacking, development of recombinant microalgae based on metabolic engineering has been limited compared to work in higher plants. Although foreign gene expression in a few microalgae species has been achieved, the relatively lower expression level makes it imperative to develop effective industrial scale microalgae bioreactors. GPAT is the key enzyme in triglyceride biosynthetic pathway. This study focused on functional and molecular characterization of GPAT, as well as its role in enhancing the lipid accumulation in oleaginous microalgae. Overexpression of GPAT in transgenic $P$. tricornutum promoted the formation of oil bodies and stimulated twofold neutral lipid content with an overall increased productivity. These results highlighted the concept that a metabolic engineering approach could be the most effective method for achieving maximal enzymatic activity and thus maximal lipid productivity in microalgae. Using a genetic-metabolomic approach, it should be possible to successfully boost TAG storage levels in microalgae, thus increasing effective routes for biofuel production.

\section{Methods}

\section{Algal culture conditions}

The microalga strain, P. tricornutum (CCMP2561), was obtained from the National Center for Marine Algae and
Microbiota. Microalgae were grown as batch cultures in flasks containing $\mathrm{f} / 2$ medium, which was filter-sterilized through $0.22 \mu \mathrm{m}$ filters. Cultures in liquid medium or on the plate were grown at $21 \pm 1{ }^{\circ} \mathrm{C}$ in an artificial climate incubator (Ningbo, China). Cool-white fluorescent tubes provided an irradiance of $200 \mu$ mol photons $\mathrm{m}^{-2} \mathrm{~s}^{-1}$ under long-day light conditions (12/12 h light/ dark regime). Cell numbers of the cultures were counted using Brightline Hemocytometer under microscope at the same time every day, in triplicates. Cell density was calculated according to Yang et al. [26].

\section{Cloning of putative GPAT and construction of expression vector}

Genomic DNA of P. tricornutum was extracted using Universal Genomic DNA Extraction Kit Ver.3.0 (Takara, China). The full-length coding region of putative PtGPAT was cloned by PCR with primers designed according to genome sequence of $P$. tricornutum on JGI website. Primer pairs used were Pt49 (5'-ACCATGGACTT ATCTACGGTTCAATCAG-3') and Pt73 (5'-GTCTTAT TCTTGTCGGAGTCGTTG-3'). The PCR product was cloned into pHY29 derived from Yao et al. [39] by overlap extension PCR cloning method [40]. In the resultant plasmid, a c-Myc tag (amino acid sequence: EQKLISEEDL) was fused at the C-terminal PtGPAT for the detection of its protein expression.

\section{Generation of transgenic $P$. tricornutum}

Phaeodactylum tricornutum was transformed by electroporation with pHY29-GPAT following the previously developed protocol by Niu et al. [41]. The transformed P. tricornutum plates were incubated for 3 weeks under standard growth conditions and the emerging putative transformed colonies on the selection medium were counted. Survived colonies were picked and inoculated in liquid $\mathrm{f} / 2$ medium containing chloramphenicol (250 mg L ${ }^{-1}$ ), then subcultured for five more cycles on selection medium to obtain stable transgenic microalgae. Selected cells were subcultured in $\mathrm{f} / 2$ medium once a week supplemented with chloramphenicol $\left(250 \mathrm{mg} \mathrm{L}^{-1}\right)$. Cells in stationary phase were collected for molecular characterization.

To detect the integration of CAT, transformed microalgae were screened by single-cell PCR analysis with primers CAT-f and CAT-r [42]. PCR-confirmed cultures were then subjected to successive culture.

\section{Characterization of photosynthesis}

The chlorophyll fluorescence parameter $\mathrm{Fv} / \mathrm{Fm}$ (ratio of variable/maximum fluorescence), an empirically verifiable index of photosynthetic performance and acclimation status [22, 23], was used to characterize microalgal 
photosynthesis. Algal cultures were kept in the dark for at least $40 \mathrm{~min}$. The chlorophyll fluorescence in the $P$. tricornutum culture was measured with a TD-700 fluorometer (Turner Design,USA) following the protocol by Macedo et al. [43].

\section{Elemental analysis}

An aliquot of $200 \mathrm{~mL}$ transgenic and wild-type cultures $\left(5 \times 10^{7}\right.$ cells $\left.\mathrm{mL}^{-1}\right)$ were harvested by centrifugation at $4{ }^{\circ} \mathrm{C}$. The pellet was resuspended in $1 \mathrm{~mL}$ water and centrifuged at $4{ }^{\circ} \mathrm{C}$. The pellet was resuspended in $1 \mathrm{~mL}$ water and centrifuged again. The pellet was baked at $60{ }^{\circ} \mathrm{C}$ for $48 \mathrm{~h}$ and was ground into powder. The powders were analyzed by using the instrument FE-SEM (Hitachi S-4800, Japan).

\section{Lipid analysis}

Lipid content in transgenic $P$. tricornutum, transgenic microalgae with nitrogen deprivation, and wild type was measured by Nile red (Sigma, USA) staining according to Yang et al. [26]. Seven-day-old microalgae culture was collected and subcultured in $-\mathrm{N}$ and $+\mathrm{N}$ medium. For Nile red staining, $30 \mu \mathrm{L}$ of solution of Nile red $\left(0.1 \mathrm{mg} \mathrm{mL}{ }^{-1}\right.$ in acetone) was added into $3 \mathrm{~mL}$ of cell culture. Non-stained cultures were used as an auto-fluorescence control. Excitation and emission wavelength for detection were set at 530 and $580 \mathrm{~nm}$, respectively. The fluorescence intensities reflect the relative neutral lipid content of stained cells.

The neutral lipid content of microalgae was also measured by gravimetric determination. An aliquot of $200 \mathrm{~mL}$ $\left(6.55 \times 10^{7}\right.$ cells $\left.\mathrm{mL}^{-1}\right)$ of 7-day-old culture was harvested and lyophilized and the dry weight of microalgae was measured. To the lyophilized cells, $2 \mathrm{~mL}$ of methanol, $2 \mathrm{~mL}$ of chloroform, and $1 \mathrm{~mL}$ of $5 \% \mathrm{NaCl}$ were added and vortexed for $2 \mathrm{~min}$. The mixture was centrifuged at $8000 \times g$ for $4 \mathrm{~min}$, and the chloroform layer was collected. The same extraction procedure was repeated three times and the combined extracts were dried by $\mathrm{N}_{2}$ flow. The lipid residue was dried in oven at $60{ }^{\circ} \mathrm{C}$ and weighed to yield dry weight.

Lipid composition was analyzed by gas chromatography-mass spectrometry (GC-MS) according to Yang et al. [26]. Fatty acids were identified according to the equipped NBS spectrum library. The integrated peak areas were determined and calculated by normalization to obtain the percent contents of fatty acid composition.

\section{Analysis of GPAT expression by qPCR and Western blotting} The expression of the integrated GPAT from the stationary phase culture was analyzed by reverse transcriptase PCR, real-time PCR, and Western blot analysis. For Reverse Transcriptase PCR analysis, RNA was extracted from
PCR-confirmed culture and wild-type culture using Takara RNA kit (Takara, Japan). RT-PCR was carried out with HiScript $^{\circledR}$ II Q RT SuperMix for qPCR + gDNA wiper (Vazyme, China) according to manufacturer's instruction.

The expression of GPAT was quantified by quantitative real-time PCR (qPCR) using SYBR Green I PCR Master Mix (Bio-Rad, USA) on ABI PRISM ${ }^{\circledR} 7500$ Sequence Detection System (ABI, USA). PtGPAT was amplified using primers:

q49 (5'-ACGACAAGGTCGGAACAAAC-3') and q73 (5'-TAAAGGCACCGTCCTTGAAC-3'). $\beta$-actin was used as reference gene. The threshold cycle $(\mathrm{Ct})$ for each well was measured and mRNA levels of the PtGPAT were quantified after normalization to $\beta$-actin.

The expression of PtGPAT protein was examined by Western blot analysis with an anti-Myc antibody against the Myc-tagged PtGPAT. An aliquot of $200 \mathrm{~mL}$ microalgal culture $\left(5 \times 10^{7}\right.$ cells $\left.\mathrm{mL}^{-1}\right)$ was harvested and total protein was extracted with Protein Extraction Kit as per the supplier's instruction (KeyGEN, China). Protein concentration was estimated by Bradford protein assay (BioRad, USA). Proteins were separated by electrophoresis by $10 \%$ SDS-PAGE and electrotransferred to polyvinylidene fluoride membrane. The membrane was blocked with skimmed milk for $2 \mathrm{~h}$ at $4{ }^{\circ} \mathrm{C}$. Thereafter, membrane was treated with anti-Myc antibody (1:3000; Sigma-Aldrich, USA). The anti-GAPDH antibody was used as reference (1:3000; Sigma-Aldrich, USA). HRP-conjugated goat antirabbit antibody (CST, USA) at a dilution of 1:5000 was used as secondary antibody. Membrane development was performed using chemiluminescent system.

\section{Morphological observation of $P$. tricornutum and subcellular localization of GPAT}

To observe the cell morphology and oil bodies in P. tricornutum, cells were stained with Nile red fluorescence dye and observed under a fluorescence microscope. One-milliliter microalgae cells were stained with $10 \mu \mathrm{L}$ of Nile red $\left(0.1 \mathrm{mg} \mathrm{mL}^{-1}\right.$ in acetone) and incubated in darkness for 20 min. A laser-scanning confocal microscope Zeiss LSM 510meta (Zeiss, Germany) with excitation wavelength of $488 \mathrm{~nm}$ and emission wavelength of $505-550 \mathrm{~nm}$ was used to observe the morphology of the stained cells.

Subcellular localization of PtGPAT was analyzed using Target P, WoLF PSORT, and LocTree3. In order to localize the expressed PtGPAT, immune-electron microscopy was employed according to Yang et al. [26]. Transgenic and wild-type cells were centrifuged at $3000 \times g$ for $10 \mathrm{~min}$. The specimens were fixed with $2 \%$ paraformaldehyde and $2 \%$ glutaraldehyde in $0.1 \mathrm{M}$ phosphate buffer ( $\mathrm{pH}$ 7.2) under vacuum for $20 \mathrm{~min}$ and followed by incubation at room temperature for $3 \mathrm{~h}$. The steps of thin sectioning and immuno-electron microscopy were 
performed as described previously. The sections were photographed by JEM-2000EXII electron microscope (JEOL, Japan) operating at $80 \mathrm{kV}$.

\section{Abbreviations}

DAG: diacylglycerol; EM: electron microscopy; GC-MS: gas chromatographymass spectroscopy; GPAT: glycerol-3-phosphate acyltransferase; LPAAT: lysophosphatidic acid acyltransferase; LysoPA: lysophosphatidic acid; MUFA: monounsaturated fatty acid; PA: phosphatidic acid; PUFA: polyunsaturated fatty acid; SFA: saturated fatty acid; TAG: triglycerol.

\section{Authors' contributions}

YFN and XW performed the majority of the experimental analysis. DXH and DWL analyzed the data. BS, WDY, and JSL provided technical expertise and made the figures and tables. HYL conceptualized the study and wrote the manuscript. All authors discussed the results and commented on the manuscript. All authors read and approved the final manuscript.

\section{Author details}

${ }^{1}$ Key Laboratory of Eutrophication and Red Tide Prevention of Guangdong Higher Education Institutes, College of Life Science, Jinan University, Guangzhou 510632, China. ${ }^{2}$ Shenzhen Polytechnic, Shenzhen 518000, China.

\section{Acknowledgements}

This work was supported by the Natural Science Foundation of China (41576132), the Guangdong Natural Science Foundation (2014A030308010), and the Department of Education of Guangdong (2014KTSCX022).

\section{Competing interests}

The authors declare that they have no competing interests.

Received: 4 June 2015 Accepted: 2 March 2016

Published online: 12 March 2016

\section{References}

1. Rawat I, Kumar RR, Mutanda T, Bux F. Biodiesel from microalgae: a critical evaluation from laboratory to large scale production. Appl Energy. 2013;103:444-67.

2. Hu Q, Sommerfeld M, Jarvis E, Ghirardi M, Posewitz M, Seibert M, et al. Microalgal triacylglycerols as feedstocks for biofuel production: perspectives and advances. Plant J. 2008:54:621-39.

3. Williams N. New biofuel questions. Curr Biol. 2010;20:R219-20.

4. Radakovits R, Jinkerson RE, Darzins A, Posewitz MC. Genetic engineering of algae for enhanced biofuel production. Eukaryot Cell. 2010;9:486-501.

5. Misra N, Panda PK. In search of actionable targets for agrigenomics and microalgal biofuel production: sequence-structural diversity studies on algal and higher plants with a focus on GPAT protein. Omics. 2013:17:173-86.

6. Haslam RP, Ruiz-Lopez N, Eastmond P, Moloney M, Sayanova O, Napier JA. The modification of plant oil composition via metabolic engineeringbetter nutrition by design. Plant Biotechnol J. 2013;11:157-68.

7. Nagle CA, An J, Shiota M, Torres TP, Cline GW, Liu ZX, et al. Hepatic overexpression of glycerol-sn-3-phosphate acyltransferase 1 in rats causes insulin resistance. J Biol Chem. 2007;282:14807-15.

8. Courchesne NMD, Parisien A, Wang B, Lan CQ. Enhancement of lipid production using biochemical, genetic and transcription factor engineering approaches. J Biotechnol. 2009;141:31-41.

9. Fan J, Andre C, Xu C. A chloroplast pathway for the de novo biosynthesis of triacylglycerol in Chlamydomonas reinhardtii. FEBS Lett. 2011;585:1985-91.

10. Nagle CA, Vergnes L, DeJong H, Wang S, Lewin TM, Reue K, et al. Identification of a novel sn-glycerol-3-phosphate acyltransferase isoform, GPAT4, as the enzyme deficient in Agpat6-/-mice. J Lipid Res. 2008;49:823-31.

11. Yet SF, Lee S, Hahm YT, Sul HS. Expression and identification of p90 as the murine mitochondrial glycerol-3-phosphate acyltransferase. Biochemistry. 1993;32:9486-91.
12. Bhat BG, Wang P, Kim J-H, Black TM, Lewin TM, Fiedorek FT, et al. Rat snglycerol-3-phosphate acyltransferase: molecular cloning and characterization of the CDNA and expressed protein. BBA-Mol Cell Biol Lipids. 1999;1439:415-23.

13. Lu B, Jiang YJ, Kim P, Moser A, Elias PM, Grunfeld C, et al. Expression and regulation of GPAT isoforms in cultured human keratinocytes and rodent epidermis. J Lipid Res. 2010;51:3207-16.

14. Zheng Z, Xia Q, Dauk M, Shen W, Selvaraj G, Zou J. Arabidopsis AtGPAT1, a member of the membrane-bound glycerol-3-phosphate acyltransferase gene family, is essential for tapetum differentiation and male fertility. Plant Cell. 2003;15:1872-87.

15. Yang W, Simpson JP, Li-Beisson Y, Beisson F, Pollard M, Ohlrogge JB. A land-plant-specific glycerol-3-phosphate acyltransferase family in Arabidopsis: substrate specificity, sn-2 preference, and evolution. Plant Physiol. 2012;160:638-52.

16. Nishida I, Tasaka Y, Shiraishi H, Murata N. The gene and the RNA for the precursor to the plastid-located glycerol-3-phosphate acyltransferase of Arabidopsis thaliana. Plant Mol Biol. 1993;21:267-77.

17. Gidda SK, Shockey JM, Rothstein SJ, Dyer JM, Mullen RT. Arabidopsis thaliana GPAT8 and GPAT9 are localized to the ER and possess distinct ER retrieval signals: functional divergence of the dilysine ER retrieval motif in plant cells. Plant Physiol Biochem. 2009;47:867-79.

18. Daboussi F, Leduc S, Maréchal A, Dubois G, Guyot V, Perez-Michaut C, et al. Genome engineering empowers the diatom Phaeodactylum tricornutum for biotechnology. Nat Comm. 2014;5:3831.

19. Niu YF, Zhang MH, Li DW, Yang WD, Liu JS, Bai WB, et al. Improvement of neutral lipid and polyunsaturated fatty acid biosynthesis by overexpressing a type 2 diacylglycerol acyltransferase in marine diatom Phaeodactylum tricornutum. Mar Drugs. 2013;11:4558-69.

20. Gong Y, Zhang J, Guo X, Wan X, Liang Z, Hu CJ, et al. Identification and characterization of PtDGAT2B, an acyltransferase of the DGAT2 acyl-Coenzyme A: diacylglycerol acyltransferase family in the diatom Phaeodactylum tricornutum. FEBS Lett. 2013;587:481-7.

21. Cui Y, Zheng G, Li X, Lin H, Jiang P, Qin S. Cloning and characterization of a novel diacylglycerol acyltransferase from the diatom Phaeodactylum tricornutum. J Appl Phycol. 2013;25:1509-12.

22. Campbell D, Hurry V, Clarke A, Gustafsson P, Oquist G. Chlorophyll fluorescence analysis of cyanobacterial photosynthesis and acclimation. Microbiol Mol Biol Rev. 1998;62:667-83.

23. Lee KM, DaSilva NA. Evaluation of the Saccharomyces cerevisiae ADH2 promoter for protein synthesis. Yeast. 2005;22:431-40.

24. Nishida I, Murata N. Chilling sensitivity in plants and cyanobacteria: the crucial contribution of membrane lipids. Annu Rev Plant Biol. 1996;47:541-68.

25. Abida H, Dolch LJ, Meï C, Villanova V, Conte M, Block MA, et al. Membrane glycerolipid remodeling triggered by nitrogen and phosphorus starvation in Phaeodacty/um tricornutum. Plant Physiol. 2015;167:118-36.

26. Yang ZK, Niu YF, Ma YH, Xue J, Zhang MH, Yang WD, et al. Molecular and cellular mechanisms of neutral lipid accumulation in diatom following nitrogen deprivation. Biotechnol Biofuels. 2013;6:67.

27. Grima EM, Medina AR, Giménez AG, González MJI. Gram-scale purification of eicosapentaenoic acid (EPA,20,5n-3) from wet Phaeodactylum tricornutum UTEX 640 biomass. J Appl Phycol. 1996;8:359-67.

28. Yokoi S, Higashi SI, Kishitani S, Murata N, Toriyama K. Introduction of the cDNA for shape Arabidopsis glycerol-3-phosphate acyltransferase (GPAT) confers unsaturation of fatty acids and chilling tolerance of photosynthesis on rice. Mol Breeding. 1998;4:269-75.

29. Liu F, Xia Y, Wu L, Fu D, Hayward A, Luo J, et al. Enhanced seed oil content by overexpressing genes related to triacylglyceride synthesis. Gene. 2015;557:163-71.

30. Iskandarov U, Sitnik S, Shtaida N, Didi-Cohen S, Leu S, Khozin-Goldberg I, et al. Cloning and characterization of a GPAT-like gene from the microalga Lobosphaera incisa (Trebouxiophyceae): overexpression in Chlamydomonas reinhardtii enhances TAG production. J Appl Phycol. 2015:1-13.

31. Payá-Milans M, Venegas-Calerón M, Salas JJ, Garcés R, Martínez-Force E. Cloning, heterologous expression and biochemical characterization of plastidial sn-glycerol-3-phosphate acyltransferase from Helianthus annuus. Phytochemistry. 2015;111:27-36.

32. Ehrlich JS, Hansen MD, Nelson WJ. Spatio-temporal regulation of Rac1 localization and lamellipodia dynamics during epithelial cell-cell adhesion. Dev Cell. 2002;3:259-70. 
33. Ishizaki O, Nishida I, Agata K, Eguchi G, Murata N. Cloning and nucleotide sequence of cDNA for the plastid glycerol-3-phosphate acyltransferase from squash. FEBS Lett. 1988;238:424-30.

34. Ouyang LL, Chen SH, Li Y, Zhou ZG. Transcriptome analysis reveals unique C4-like photosynthesis and oil body formation in an arachidonic acid-rich microalga Myrmecia incisa Reisigl H4301. BMC Genom. 2013;14:396.

35. Gidda SK, Shockey JM, Falcone M, Kim PK, Rothstein SJ, Andrews DW, et al. Hydrophobic-domain-dependent protein-protein interactions mediate the localization of GPAT enzymes to ER subdomains. Traffic. 2011;12:452-72.

36. Rizzo AM, Prussi M, Bettucci L, Libelli IM, Chiaramonti D. Characterization of microalga Chlorella as a fuel and its thermogravimetric behavior. Appl Energ. 2013;102:24-31.

37. Klok AJ, Lamers PP, Martens DE, Draaisma RB, Wijffels RH. Edible oils from microalgae: insights in TAG accumulation. Trends Biotechnol. 2014;32:521-8.

38. Merchant SS, Kropat J, Liu B, Shaw J, Warakanont J. TAG, You're it! Chlamydomonas as a reference organism for understanding algal triacylglycerol accumulation. Curr Opin Biotech. 2012;23:352-63.
39. Yao Y, Lu Y, Peng K-T, Huang T, Niu YF, Xie WH, et al. Glycerol and neutral lipid production in the oleaginous marine diatom Phaeodactylum tricornutum promoted by overexpression of glycerol-3-phosphate dehydrogenase. Biotechnol Biofuels. 2014;7:110.

40. Bryksin A, Matsumura I. Overlap extension PCR cloning: a simple and reliable way to create recombinant plasmids. Biotechniques. 2010;48:463-5.

41. Niu YF, Yang ZK, Zhang MH, Zhu CC, Yang WD, Liu JS, et al. Transformation of diatom Phaeodactylum tricornutum by electroporation and establishment of inducible selection marker. Biotechniques. 2012; doi:10.2144/000113881.

42. Xue J, Niu YF, Huang T, Yang WD, Liu JS, Li HY. Genetic improvement of the microalga Phaeodactylum tricornutum for boosting neutral lipid accumulation. Metab Eng. 2015;27:1-9.

43. Macedo R, Lombardi A, Omachi C, Rörig L. Effects of the herbicide bentazon on growth and photosystem II maximum quantum yield of the marine diatom Skeletonema costatum. Toxicol In Vitro. 2008;22:716-22.

\section{Submit your next manuscript to BioMed Central and we will help you at every step:}

- We accept pre-submission inquiries

- Our selector tool helps you to find the most relevant journal

- We provide round the clock customer support

- Convenient online submission

- Thorough peer review

- Inclusion in PubMed and all major indexing services

- Maximum visibility for your research

Submit your manuscript at www.biomedcentral.com/submit
(O) Biomed Central 